Published in final edited form as:

Barbetta, A., Bertinetti, L., \& Zemb, T. (2017). Composition dependent Equation of State of Cellulose Based Plant Tissues in the presence of Electrolytes. Colloids and Surfaces A: Physicochemical and Engineering Aspects, 532, 314-322. doi:10.1016/j.colsurfa.2017.04.075.

\title{
Composition dependent Equation of State of Cellulose Based Plant Tissues in the presence of Electrolytes
}

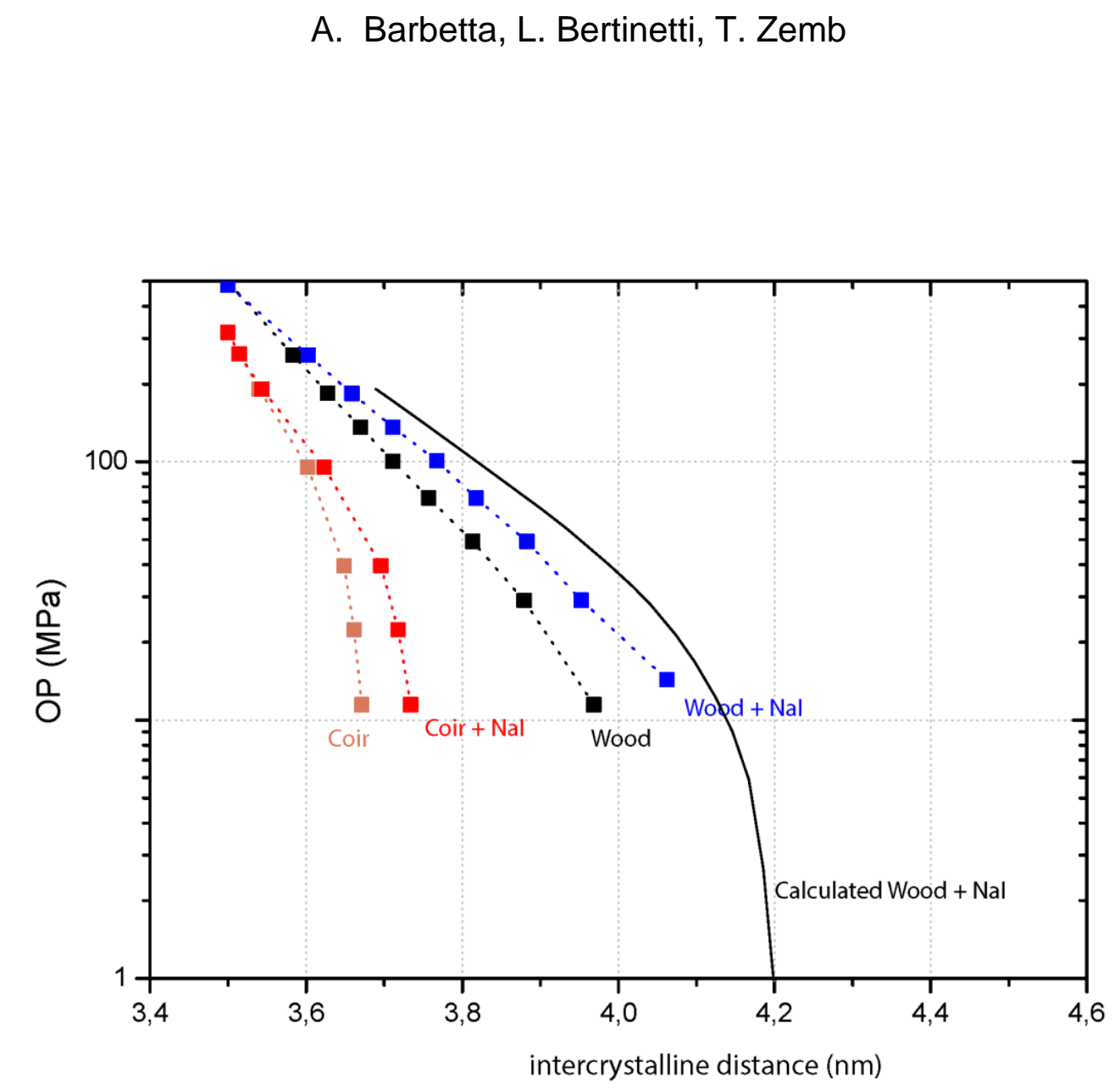




\section{Highlights}

- Wood cell wall Equation of State, calculated from modeling, is compared to classical models based on fitting of experimental sorption parameters

- The addition of an electrostatic term to the Equation of State extends its validity to the case of impregnated electrolytes

- Gravimetric experimental wood Equation of State shows an acceptable correspondence with the calculated curve

- Wood hemicelluloses play a dominant role during electrolytes adsorption

Cell walls of so-called "wood-materials" are constituted by a complex, highly anisotropic and hierarchically organized nanocomposite, characterized by stiff crystalline cellulose nano-fibers, parallel to each other, and embedded in a softer and less anisotropic matrix of hemicelluloses, lignin and water. This matrix is hygroscopic, and therefore swells with increasing humidity. Consequently, wood cells undergo large dimensional changes. A minimal model of wood secondary cell walls to predict water absorption has recently been developed by Bertinetti and co-workers ${ }^{[1]}$ in the form of an Equation of State (EOS) that represents equivalently the water sorption versus relative humidity, as considered in chemical engineering, or the relation between osmotic pressure and volume of solutes, in the physical chemistry equation of state approach initiated by Jean Perrin. We extend hereby this model to the presence of electrolytes adsorbed in the gel part wood cell wall and compare compression wood cell walls to the extreme case of coir.

Keywords: wood cell walls; impregnation; salt; swelling; equation of state

\section{Introduction}

Wood cell wall is a nanocomposite material. ${ }^{[2,3]}$ It is constituted by parallel stiff crystalline cellulose fibers (ca. 50\%), embedded in a soft matrix composed by hemicelluloses (20-35\%), lignin (10-25\%) and water. ${ }^{[4]}$ This matrix is hygroscopic, and undergoes dimensional changes (i.e. anisotropic 
swelling) upon water uptake, or upon increasing relative humidity. This swelling behavior can be expressed in terms of Equation of State (EOS) as it has been recently established. ${ }^{[1]}$ Equations of state can be expressed either using quantities typical of chemical and materials engineering, i.e. the water content versus relative humidity, or in quantities used in physical chemistry, i.e. the osmotic pressure, seen as a chemical potential of water divided by the molar volume ${ }^{[5]}$ versus a distance, or a volume, ${ }^{[6]}$ as introduced by Jean Perrin using monodisperse emulsions of latex in his Nobel prize winning work, early in the XXth century. ${ }^{[7]}$

As the EOS, depending on the field, can be expressed using several different units, in Appendix A we present the relationships between the physical quantities needed to convert the EOSs in the different languages:

- the universal relation between relative humidity and osmotic pressure;

- the relation between water uptake and inter-crystalline distance $d$ in the case of compression wood cell walls and for a specific cellulose crystal size;

- $\quad$ the relation between spacing $d^{*}$ and the water volume fraction (water volume versus total volume) as well as the crystalline cellulose volume fraction knowing that wood cell wall is comprised of four main components of known volumes (crystalline cellulose, soluble cellulose, lignin and water).

The aim of the present work is to extend that Equation of State to the case of cellulose-based materials containing crystalline cellulose in the presence of electrolytes. The chosen reference model system is spruce compression wood either as is for the standard EOS, or impregnated for a long period with a concentrated electrolytes solution to extend the EOS taking into account electrostatic forces.

An important "excess" swelling is sometimes observed if wood is impregnated with a solution containing electrolytes. This is a specific effect: larger strains are induced by so-called "antagonistic 
salts", ${ }^{[8]}$ made by one ion that can easily lose its hydration sphere ("chaotrope") and adsorb at the interface between cell walls constituents, and a counter-ion that stays in the space between them, in its hydrated form ("cosmotrope"). The case of maximal swelling has been considered in a previous work, ${ }^{[9]}$ and indicates the asymptotic limit of the EOS at zero osmotic stress.

Efficient swelling agents are for example $\mathrm{NaI}$ and $\mathrm{CaBr}_{2}$ : axial strains along the fibers direction $\varepsilon_{x}$ of respectively ca. $3 \%$ and $2.5 \%$ are measured with a tensile stage on thin spruce compression wood foils $(150 \mu \mathrm{m}$ thick) when changing the sample environment from water to the salt solution.

Specific effects also occur in the case of samples pre-treated with salt solutions impregnation and afterwards exposed to decreasing relative humidity, $\mathrm{RH}$, (i.e. increasing the osmotic pressure, OP), as shown recently using Environmental Scanning Electron Microscopy (ESEM) ${ }^{[10]}$. Dehydration experiments were performed via ESEM in controlled RH conditions: the processing of the recorded images allowed to track clear details in material structure and evaluate the relative changes in their distance during drying. Results showed an axial strain $\varepsilon_{x}$ of $-3 \%$ from $100 \% \mathrm{RH}$ to $25 \% \mathrm{RH}$ in the case of samples pretreated with a NaI solution, compared with an $\varepsilon_{x}$ of $-2.45 \%$ in the same $\mathrm{RH}$ range in the case of non pre-treated samples. Although these effects can be measured only in relative terms with most of the classical techniques used in the field, with an appropriate knowledge of material's structure, by means of scattering techniques it is possible to reinterpret these data in terms of absolute values: with small angle X-ray scattering (SAXS), for example, it is possible to evaluate the absolute distance between adjacent cellulose crystals, which has been shown to be 4.25 $\mathrm{nm}$ and $4.04 \mathrm{~nm}$, respectively for the samples pretreated with $\mathrm{NaI}$ and the ones that were not pretreated. ${ }^{[9]}$

Previously to the abovementioned combined microscopy and X-ray micro-investigations, ${ }^{[11]}$ values of wood moisture content were obtained via different techniques, such as gravimetric methods, based on measures of the weight difference between wet and oven dried samples, ${ }^{[12]}$ or using distillation devices, in which liquids immiscible with water that are good solvents for wood volatile 
components avoid experimental errors due to evaporation. ${ }^{[13]}$ Lately, more modern instruments that give faster and more automatic responses were applied for calculations of MC, such as NMR ${ }^{[14]}$ or electrical moisture meters. ${ }^{[15]}$

Starting from experiment performed with these techniques, more than 70 different general models have been developed in chemical engineering as parametric expressions to fit sorption isotherm data, and have been applied to water uptake by wood. ${ }^{[16]}$ Most common models are either based on considering water adsorption at a surface or considering sorption as a solution phenomenon. Although the hypotheses underlying their formulation is different, they are mathematically equivalent. ${ }^{[17]}$

Examples of the first type are BET ${ }^{[18]}$ and Dent ${ }^{[19]}$ models, whose constituent equations are derived by considering the equilibrium of condensation and evaporation of water on wood cell wall sorption sites (i.e. hydroxyls) with different binding energies for each binding layer.

In the most popular approach of the second kind, as in the Hailwood-Horrobin parametric model, ${ }^{[20]}$ sorbed water is considered to combine with soluble polymeric units to form a hydrate and to form a solid solution in the cell walls. Therefore, the two equilibria that are taken into account are the ones of dissolved water, with water vapor and with water molecules bounded to the polymers. The mathematical expressions for these three models are recalled in Appendix B.

In order to evaluate the quality and the reliability of the models, predicted and measured heats of sorption can be compared. Results discrepancy is around $50 \% .{ }^{[21]}$ Despite the fact that fitting the results with non-linear regression techniques could drastically reduces the difference, ${ }^{[21]}$ the gap between theory and experiments looks still remarkable at this point, especially considering that none of the models takes into account wood materials' specific structure and composition.

Moreover, at the current level a limiting factor for the theory is that matrix components' individual contributions to swelling are not yet distinguishable. Our aim in this work is to compare EOS of 
compression wood with the ones obtained for another cellulosic natural materials with different composition: lignin-rich poorly swelling coir fibers extracted from coconut, that are largely used since centuries in hot and wet conditions, and that are composed by cellulose (ca. 50\%) and lignin (ca. $45 \%)$.

\section{Materials and methods}

\subsection{Compression wood}

Compression wood samples from Spruce tree (Picea abies) were cut in $100 \mu \mathrm{m}$ slices, in the parallel direction with respect to fibers orientation, with a Leica-rm2255 microtome, stored in freezer at $-20^{\circ} \mathrm{C}$, to preserve their natural conditions, and unfrozen in a fridge at $4^{\circ} \mathrm{C}$ the day before the experiments.

\section{$\underline{2.2 \text { Coir }}$}

Coir fibres, extracted from the husk of coconut (Cocos nucifera), were obtained from from Hayleys (Sri Lanka), that produces them for fiber mattresses, and stored in dry ambient conditions.

\section{$\underline{2.3 \text { Chemicals }}$}

Sodium iodide solutions were prepared with Alfa Aesar salt just before the experiments. 15.2 osm concentration was chosen according to previous experiments [9], and it produces the same osmotic pressure of a saturated $\mathrm{NaCl}$ solution $(37.7 \mathrm{MPa})$, that has been already used as a reference in the previous works. ${ }^{[9]}$

\section{$\underline{2.4 \text { DSC measurements }}$}

Gravimetric EOS were elaborated from DSC measurements, performed with a SENSYS evo DSC from Setaram Inc. Samples were cut into small slices for an overall weight of ca. $30 \mathrm{mg}$, and once 
the instrument was tared they were introduced in the crucibles for equilibration. The initial RH\% point was set according to the environmental condition to reduce the uncontrolled changes that could occur to the sample before the starting of the measurements. The experiments of hydration consisted on multiple steps at different $\mathrm{RH} \%$, from dry (4\%RH) to wet (92\%RH). Step times were longer (5h) for the first points, that requires longer period for equilibration, than for the others (3h).

\subsection{Force balance method for the case of swelling in the absence of impregnating electrolytes}

Rationalization of the process of water uptake by wood starts from a geometric minimal model of wood cell walls, at nanometric scale, in which the hexagonal cellulose crystals are considered as coated by a thin layer of a homogeneous matrix, as the distribution of its components in the polymeric gel are not yet fully understood..$^{[1]}$

In any equation of state approach molecular and macroscopic forces simultaneously contributing to wood spatial deformations (either by favoring or limiting swelling) are individually evaluated and then summed up in the form of a force balance, in which derivatives of chemical, colloidal and mechanical energies are calculated. ${ }^{[22]}$ The force balance developed so far for cell walls includes: enthalpy of chemical binding between cellulose fibers and matrix components, dependent on the number of contact points, free energy of hydration repelling cellulose crystals separated by a layer of water, configurational entropy of the matrix, van der Waals force between crystals, and elastic energy generated by anisotropic swelling of the material.

The validity of the EOS can be extended to the case of wood impregnation with electrolytes, used as swelling agents, by considering also in the force balance an extra electrostatic term. If an electrolyte penetrates the gel in between the crystallites, and it is in equilibrium with excess brine, no extra term has to be considered, since electrolyte activity is the same inside the wood cell wall and outside, i.e. in the bathing fluid as well as in the large macroscopic channels present in wood cell 
wall. ${ }^{[23]}$ However, if the anion or the cation is adsorbed or complexed on the surface of the hydrophobic crystalline material, charge separation occurs: electrostatic repulsion between charged crystals is screened by the diffuse layer of counter-ions and any other "background" salt present. [24]

\section{Results and discussion}

\section{$\underline{3.1 \text { Modification of the Equation of State induced by impregnated salts }}$}

The mathematical derivation of the osmotic pressure $\Pi(D)$ considers two adjacent cellulose crystals, coated by a matrix layer, as two infinite parallel plans, separated by a water layer of a certain thickness $D$, in osmotic equilibrium with a salt solution, used as reservoir. At this level, all ions are considered to be monovalent. In the "weak overlap approximation" case (i.e. $D \gg \lambda$, where $\lambda$ is the Debye length), the layers diffusing in the proximity of the two plans weakly superpose, and the interaction is connected to the electrostatic properties in the proximity of an isolated plan. Analytical expression of the simultaneous equations to calculate the osmotic pressure is reported in

\section{Appendix C.}

The relationship between binding constant of the ions to the available sorption sites and the free energy of binding is based on a simple equation (Equation 1):

$K=\Sigma \delta e^{-\Delta G_{s} / R T}$ (Equation 1)

In this expression, $K$ is the binding constant of the binding ion, assumed to be by three orders of magnitude higher than the one of the non-binding one, $\Sigma$ is the surface area of the binding sites, taken as $1 \mathrm{~nm}^{2}, \delta$ is the diameter of the dominant binding ion, expressed in $\mathrm{nm}$, and $\Delta G_{S}$ is the binding energy in J/mol. Figure 1 shows the calculated Equation of State for three different ionic radii, corresponding to the one used in previous work $(0.200 \mathrm{~nm}$, approximately corresponding to the radius of $\left.\mathrm{I}^{-}\right)$, and the ones of $\mathrm{OH}^{-}(0.133 \mathrm{~nm})$ and of $\mathrm{Mg}^{2+}(0.072 \mathrm{~nm})$. This last one was chosen 
because, according to the theory, in the case of salts composed by monovalent anions and divalent cations (e.g. $\mathrm{MgBr}_{2}, \mathrm{CaBr}_{2}$, ecc..,) these last one are supposed to adsorb on the matrix structure via bidentate complexation. However, we stress out that, for model simplicity, all ions are treated as monovalent (i.e. valence of ion $i Z_{i}= \pm 1$ in the calculations). At a given binding constant $(K=0.035$ $\mathrm{M}^{-1}$ ), the charge separation created by selective sorption of one of the two ions leads to higher repulsive forces with increasing ionic sizes, and therefore to higher values of the maximum swelling $D_{\max }$, calculated at $100 \%$ relative humidity. Moreover, the sensitivity to shrinkage with decreasing relative humidity (i.e. increasing $\Pi$ ), looks higher for smaller ions. Results of variations of free energy of adsorption (in the model, this means a tuning of the binding constant) at a fixed ionic radius for $\delta=0.2 \mathrm{~nm}$ are reported in Figure 2:

Results show an evident increase of the maximum swelling $D_{\max }$ at small values of binding constant (higher $\Delta G_{S}$ ), until the point at which charges are saturated and it reaches its maximum value: $D_{\max }=4.50 \mathrm{~nm}$ at $K=0.035 \mathrm{M}^{-1}$. At higher values (i.e. with decreasing free energy of binding), charge screening leads to a slight shrinkage, with a resulting $\Delta D_{\max }$ of $-0.02 \mathrm{~nm}$ in a range of $K$ of more than one order of magnitude. Results of the theoretical curve can be compared with experimental results published in literature ${ }^{[9]}$ for $D_{\max }$ in the case of compression wood impregnated with a $4.70 \mathrm{M} \mathrm{NaI}$ solution, that indicate weak ion binding with positive values of $\Delta G_{S}$ $(8 \mathrm{~kJ} / \mathrm{mol})$.

\subsection{Comparing the swelling observed with results by fitting to parametric sorption models}

The EOS based on first principle of colloidal forces can be compared with the EOS calculated from first principles with parametric water sorption isotherms such as general BET, ${ }^{[18]}$ or models adapted to cellulosic materials such as the very popular ones initially proposed by Dent, ${ }^{[19]}$ HailwoodHorrobin. ${ }^{[20]}$ In BET and Dent models sorption is considered as a surface phenomenon, in which 
water is sorbed on wood cell wall sorption sites, and supposed forming different "layers" in the crystalline cellulose. Dent model can be considered as modification of generic BET equations, in which secondary layers have a specific binding energy and constant which is assumed not to be the same as liquid water, as in the BET model.

On the other hand, in the Hailwood-Horrobin $(\mathrm{H}-\mathrm{H})$ theory sorption is considered as a solution phenomenon: sorbed water forms an "hydrate" with the wood, and a solid solution is formed in the cell wall. In this case, the two equilibria defining the model equation are the ones of the dissolved water with the environmental water vapor and with the hydrated water.

Wood EOS, predicted from the model, is hereby compared from result obtained from classical sorption theories, such as BET, Dent and Hailwood-Horrobin $(\mathrm{H}-\mathrm{H})$. Derivation of the used equations is explained in details in Appendix B. Figure 3a compares the Equation of State derived from modeling with the classical aforementioned models. The three models strongly depend on three input parameters: the moisture content in hydrated conditions, and on the equilibrium constants of water condensation and evaporation. Used parameters for BET, Dent and $\mathrm{H}-\mathrm{H}$ equations are also reported in the supplementary information (Table B.1). BET curve strongly differs from the model curve, while Dent and $\mathrm{H}-\mathrm{H}$, which mathematically correspond, give more consistent results. It is important to point out that these theories have limited applicability, as swelling is not taken into account. Nevertheless, non-linear regression techniques can be used to fit these results to the modeling curve, as shown in Figure 3b. BET model looks clearly inadequate, while the curve representing Dent and $\mathrm{H}-\mathrm{H}$ sorption models perfectly superpose to the modeling curve. Output parameters of the fit give a binding constant for the primary sorbed water layer $K_{1}^{\prime}$ (Dent theory) of $2.4 \mathrm{M}^{-1}$, that approximately corresponds to one half of the one found in literature for spruce wood, ${ }^{[1]}$ while the constant for secondary water layers, $K_{2}^{\prime}$, do not change significantly. On the other hand, the value of the moisture content in hydrated conditions, $M_{0}^{\prime}$, is increased from $8.7 \%$ to $12.8 \%$. 


\section{$\underline{3.3 \text { Variations of the EOS with material composition }}$}

Experimental gravimetric EOS was obtained for wood via microgravimetric measurements, and compared to the one previously calculated by the model. Similarly to the case of results obtained from experiments performed via a tensile stage operating at controlled humidity, the model at its current state predicts higher water uptake than the observed one. This over-estimation was previously quantified, and data are consistent with the lower limit of the model, that has been obtained by multiplying all the attractive terms by a factor of 1.5 , and dividing all the repulsive terms by the same factor. Acquired sorption data for coir fibers are also reported, with the interest of understanding how the components of the matrix, which were so far considered as undistinguishable, individually contribute to water sorption process (Figure 4). It must be noted that, for limits of the experimental setup, the materials' weight at the lowest humidity reached (2.7 $\mathrm{RH} \%$ ) was taken as the value of dry reference to calculate the moisture content from DSC raw data.

The sorption behavior of coir material (light brown) show a slight increase of fibers' weight, with a maximum moisture content value of $7 \%$ at high humidity, approximately corresponding to one third of the one of compression wood in the same conditions, meaning a limited availability for water uptake.

In order to evaluate the effects of the impregnation with a swelling agent, dehydration experiments were repeated on compression wood and coir samples pre-treated with a $4.7 \mathrm{NaI}$ solution. Results are shown in Figure 5a. In both wood and coir fibers, salt impregnation implies a clear enhancing of water uptake process. This is more evident in the case of wood, where an increase of $10 \%$ on the moisture content at the highest humidity is observed with respect to the untreated sample. In the case of coir, the effect of impregnation is limited to an extra $4 \%$. The study of the effects of NaI impregnation on coir fibers is of primary interest in order to understand the role of each component of wood cell walls material during water uptake. On Figure $\mathbf{5 b}$ experimental results of wetting and impregnation tests, performed with a tensile stage on coir fibers, are reported: sample in dry 
conditions was at first immersed in milli-Q water, then in a $4.7 \mathrm{NaI}$, and finally washed again with water. This protocol was already used for similar experiments on compression wood samples.

Similarly to the case of wood, wetting process induces an axial elongation $\varepsilon_{\mathrm{x}}$ of ca. $3 \%$. The observed time needed for reaching the equilibrium is quite fast in the case of coir (ca. 3 hours). After one cycle of impregnation and washing, the final registered $\varepsilon_{\mathrm{x}}$ is less than $0.2 \%$, approximately $1 / 10$ of what was registered in the case of compression wood. Limited swelling indicates a weak electrolyte adsorption, meaning that hemicelluloses, abundant in wood cell wall materials but present only in traces in coir fibers, play a dominant role in the process, by furnishing the terminal hydroxyl groups available for the adsorption of the chaotropic ions. The values of axial elongation of dry samples immersed in water before electrolyte adsorption, that are similar for both materials, indicates that the two processes are independent.

\section{Conclusion}

The validity of the model describing water uptake by wood cell wall material has been extended in the present work to the case of the presence of monovalent ions in the reservoir solution.

An electrostatic term due to salt impregnation has been calculated and added to the force balance, using the swelling agent $\mathrm{NaI} 4.70 \mathrm{M}$ as antagonistic salt, with a chaotropic anion adsorbing on the crystalline cellulose. Free energy of binding of the anion is typically of the order of $8 \mathrm{~kJ} / \mathrm{mol}$. Results have been reported in the form of an Equation of State (EOS), showing material's swelling and shrinkage, in terms of absolute distance between adjacent cellulose crystal fibers, as a function of environmental relative humidity. The model shows good response to variation of the equilibrium constant (i.e. free energy of adsorption) and hydrodynamic size of the binding ion. Higher ionic 
radii imply higher structure swelling, while higher binding constants lead to charge screening and therefore to a slight shrinkage.

The peculiarity of the presented model is to take material's structure as a starting point, with respect to the existing sorption models, based on experimental sorption isotherm parameters. Calculated Equations of State derived from three of these "classical" models has been derived from literature, and compared to the modeling curve: although BET result are clearly divergent, Dent and Hailwood-Horrobin data set (which are mathematically equivalent) show only a slight discrepancy at higher humidity values, and their constitutive equation parameters can be fitted to the modeling curve, until they are superposed, by halving the binding constant and increasing the moisture content at fully hydrated status of few percentage points.

It must be pointed out that, at the current state of the model, a detectable difference can still be noted in comparison with experimental results. We showed that experimental gravimetric EOS is comparable to the one derived from lower limit of the model, obtained by overestimating the attractive terms, opposing to swelling, and underestimating the repulsive ones.

In the model, wood has been considered as constituted by inert cellulose fibers embedded in a homogeneous swellable matrix. As this is normally composed of hemicelluloses and lignin, future developing of the modeling concern the evaluation of the individual contributes of the matrix components to swelling. With this goal, experimental gravimetric EOS have been studied for coir material. Coir is made of cellulose and lignin, and present a considerable electrolytic content. Coir water uptake look limited, with a maximum measured moisture content of ca. $6 \%$ at high humidity. Moreover, tensile test experiments were performed to evaluated coir fibers elongation during water and $\mathrm{NaI}$ solution uptake: while the $3 \%$ axial strain registered while passing from dry to wet sample is equal to that of compression wood, immersion in concentrated salt solution results in a negligible swelling. This favors the idea of a dominant role of hemicelluloses during electrolytes adsorption. 


\section{Acknowledgements:}

This work was supported by a PhD Grant for the Labex "Chemisyst" ANR 2011-05 centered in Montpellier, and was performed with the CNS/INC and MPG/MPIKG agreement L.I.A. "RECYCLING" 2013-2017. The authors acknowledge input for the calculation of electrostatic term of Luc Belloni as well as inputs of Helmut Möhwald, supervisor of the associated French-German $\mathrm{PhD}$.

\section{Appendixes}

\section{$\underline{\text { A. Conversion graphs }}$}

The following graphs show:

1- The correlation between relative humidity and osmotic pressure

2- The correlation between inter-crystalline distance and moisture content

3- The correlation between crystalline cellulose volume fraction and extra swelling due to the presence of salts

Experimental EOS are reported in the form showing osmotic pressure OP as a function of the distance between cellulose crystals, for the case of coir (brown), pre-treated coir with 4.70 M NaI solution (red), compression wood (blue) and pre-treated compression wood with a $4.70 \mathrm{M} \mathrm{NaI}$ solution (blue). 


\section{$\underline{\text { B. Mathematical expression of classical sorption theories equations }}$}

BET and Dent equation are defined from the equilibrium of condensation and evaporation of water from sorption sites, at each layer. The difference between the two models is in the fact that the first one approximates the thermodynamic properties of the water in the secondary layers to the one of liquid water, while in the second one specific binding constants are introduced. In terms of moisture content and relative humidity, the two resulting equations can be rearranged as follows (Equation

\section{B.1 and Equation B.2):}

BET: $M C=\frac{M_{0}^{\prime} K_{1}^{\prime} x}{(1-x)\left(1+K_{1}^{\prime} x-x\right)}$

(Equation B.1)

Dent: $M C=\frac{M_{0}^{\prime} K_{1}^{\prime} x}{\left(1-K_{2}^{\prime} x\right)\left(1+\left(K_{1}^{\prime}-K_{2}^{\prime}\right) x\right)}$

(Equation B.2)

where $x$ represents the relative humidity, reported in a scale from 0 to $1, K_{1}^{\prime}$ is equivalent to the saturated vapor pressure, multiplied by the ratio of the attachment rate constant of the vapor molecules per unit vapor pressure to the escape rate constant for the water molecules at the primary sorption. $K_{2}^{\prime}$ is similarly defined for secondary sorption sites. $M_{0}^{\prime}$ corresponds to the moisture content in the condition of complete monolayer coverage of all the available sorption sites.

The mathematical expression of the Hailwood-Horrobin model $(\mathrm{H}-\mathrm{H})$ is reported in Equation B.3:

$\mathrm{H}-\mathrm{H}: M C=\frac{M_{0} K_{2}\left(K_{1}+1\right) x}{\left(1+K_{1} K_{2} x\right)\left(1-K_{2} x\right)}$

(Equation B.3)

where the term $M_{0}$ indicates the moisture content corresponding to the condition of complete polymer hydration (i.e. one molecule of water attached to each hydratable polymer unit). The two constants $K_{1}$ and $K_{2}$ represent the equilibrium of the formation of hydrates between dissolved water and the polymeric units, and the one between the hydrated water and the water vapor, respectively. Calculated parameters for BET, Dent and H-H equations are listed in Table B.1, for spruce wood at ca. $30^{\circ} \mathrm{C}$. 


\section{Analytical derivation of the electrostatic term}

Analytic expression of osmotic pressure is reported in Equation C.1:

$\Pi=64 k T c_{s}^{\prime} \gamma^{2} e^{-\kappa D}$

(Equation C.1)

where $c_{s}^{\prime}$ is the total concentration of salt in the reservoir, intended as the sum of all the concentrations of the present ions, $\kappa=\lambda^{-1}$ is the screening constant, defined in Equation C.2:

$\kappa=\sqrt{8 \pi L_{B} c_{S}^{\prime}}$

(Equation C.2)

In this equation, $L_{B}$ represents Bjerrum length, which has a value of ca. $0.7 \mathrm{~nm}$, in water at $25^{\circ} \mathrm{C}$. Its analytical form is described in Equation C.3:

$L_{B}=\frac{e^{2}}{4 \pi \varepsilon_{0} \varepsilon k T}$

(Equation C.3)

In Equation 2, $\gamma=\tanh \left(\frac{\phi_{s}}{4}\right)$ is a coefficient related to the surface electrostatic potential $\phi_{S}$, defined from Equation C.4 (Grahame equation):

$\sin \left(\frac{\phi_{S}}{2}\right)=\frac{2 \pi L_{B} \Sigma_{S}}{\kappa}$

(Equation C.4)

where $\Sigma_{S}$ is the surface charge density.

Each ion $i$ can adsorb on surface sites via a chemical reaction, following the law of mass action, expressed in Equation C.5:

$K_{i}=\frac{[\text { site }-i]}{[\text { site }] c_{i(\text { surface })}}$

(Equation C.5)

In this expression, three terms are present: the surface concentration of sites occupied by an $i$ ion, that of the unoccupied sorption sites, and the volumetric concentration of ions $i$ in contact with the 
plan where the reaction takes place. In Equation C.6, the term $c_{i(\text { surface })}$ is explained, and the law of mass action is written as a function of the occupation rate $\alpha_{i}$ if each $i$ ion:

$K_{i}=\frac{\alpha_{i}}{\left(1-\sum_{j} \alpha_{j}\right) c_{i}^{\prime} e^{-Z_{i} \phi_{S}}}$

(Equation C.6)

where the valence $Z_{i}$ can be either +1 or -1 .

The surface charge density is derived by summing all the $i$ contributions, as shown in Equation C.7:

$\Sigma_{S}=\Sigma_{0}\left(Z_{0}+\sum_{j} \alpha_{j} Z_{j}\right)$

(Equation C.7)

Iterated solution of the three simultaneous equations (Equation C.4, Equation C.6 and Equation C.7) furnishes all the required parameters, including the coefficient $\gamma$ that is needed to calculate $\Pi$.

\section{References}

[1] Bertinetti L, Fratzl P, Zemb T (2016) Chemical, colloidal and mechanical contributions to the state of water in wood cell walls. New J. Phys. 18:083048

[2] Fratzl P, Weinkamer R (2007) Nature's hierarchical materials. Prog. Mater. Sci. 52:12631334

[3] Dunlop JWC, Fratzl P (2010) Biological composites. Annu. Rev. Mate. Res 40:1-24

[4] Altaner C, Apperley DC, Jarvis MC (2006) Spatial relationships between polymers in Sitka spruce: Proton spin-diffusion studies. Holzforschung 60:665-673

[5] Wolfe J, Bryant G (1992) Physical principles of membrane damage due to dehydration and freezing. NATO ASI series : Series H : Cell biology 62:205-224 
[6] Parsegian VA, Rand RP, Fuller NL, Rau DC (1986) Osmotic stress for the direct measurement of intermolecular forces. Method. Enzymol. 127:400-416

[7] Perrin J (1913) Les atomes. Flammarions re-edited 1991

[8] Onuki A, Okamoto R, Araki T (2011) Phase transitions in soft matter induced by selective solvation. Bull. Chem. Soc. Jpn. 84:6:569-587

[9] Barbetta A, Fratzl P, Zemb T, Bertinetti L (2016) Impregnation and swelling of wood with salts: ion specific kinetics and thermodynamics effects. Adv. Mater. Interfaces 1600437

[10] Barbetta A. Bertinetti L, Lautru J, Podor R, Zemb T (Submitted) Nano-, Meso- and Macroswelling characterization of impregnated compression wood cell walls

[11] Paris O, Li C, Siegel S, Weseloh G, Emmerling F, Riesemeier H, Erko A, Fratzl P (2007) A new experimental station for simultaneous X-ray microbeam scanning for small- and wideangle scattering and fluorescence at BESSY II. J. Appl. Cryst. 40:s466-s470

[12] Hergt HFA, Christensen GN (1965) Variable retention of water by dry wood. J. Appl. Polym. Sci. 9:2345-2361

[13] Kollmann FFP, Höckele G (1962) Kritischer Vergleich einiger Bestimmungsverfahren der Holzfeuchtigkeit. Holz Roh- Werkst 20:461-473

[14] Nanassy AJ (1973) Use of wide-line NMR for measurement of moisture content in wood. Wood Sci. 5:187-193

[15] Stamm AJ (1927) The electrical resistance of wood as a measure of its moisture content. Ind. Eng. Chem. 19:1021-1025

[16] Limousin G, Gaudet JP, Charlet L, Szenknect S, Barthès V, Krimissa M (2007) Sorption isotherms: a review on physical bases, modeling and measurement. Appl. Geochem. 22: 249-275 
[17] Boquet R, Chirife J, Iglesias HA (1980) Technical note: on the equivalence of isotherm equations. J. Fd Technol. 15:345-349

[18] Brunauer S, Emmett PH, Teller E (1938) Adsorption of gases in multimolecular layers. J. Am. Chem. Soc. 60:2:309-319

[19] Dent RW (1980) A sorption theory for gas mixtures. Polym. Eng. Sci. 20:4: 286-289

[20] Hailwood AJ, Horrobin S (1946) Absorption of water by polymers. Analysis in terms of a simple model. Trans Faraday Soc. 42B:84-92, 94-102

[21] Simpson WT (1973) Predicting equilibrium moisture content of wood by mathematical models. Wood Fiber Sci. 5:1:41-49

[22] Bertinetti L, Fischer FD, Fratzl P (2013) Physicochemical basis for water-actuated movement and stress generation in nonliving plant tissues. PRL 111:238001

[23] Merk V, Chanana M, Gaan S, Burgert I (2016) Mineralization by calcium carbonate insertion for improved flame retardancy. Holzforschung 70:9:867-876

[24] Belloni L (1998) Ionic condensation and charge renormalization in colloidal suspensions. Colloids Surf. A 140:1-3:227-243 


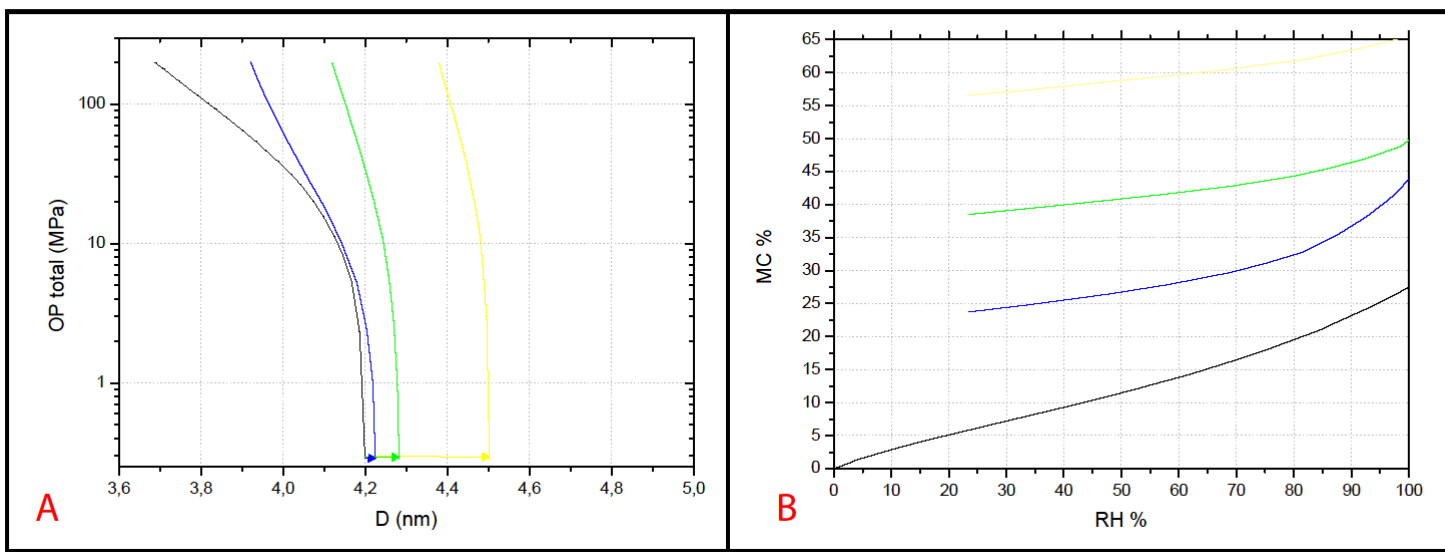

Figure 1: Calculated wood Equation of State, including electrostatic term due to the presence of impregnated salts, for three different ionic radii , corresponding to $\mathrm{Mg}^{2+}$ (blue), $\mathrm{OH}^{-}$(green) and l- (yellow), compared with the calculate EOS without salt impregnation (black). Results are reported in two languages A) Osmotic pressure vs. intercrystalline distance B) moisture content vs. relative humidity. Arrows indicate the maximum swelling $D_{\max }$. 


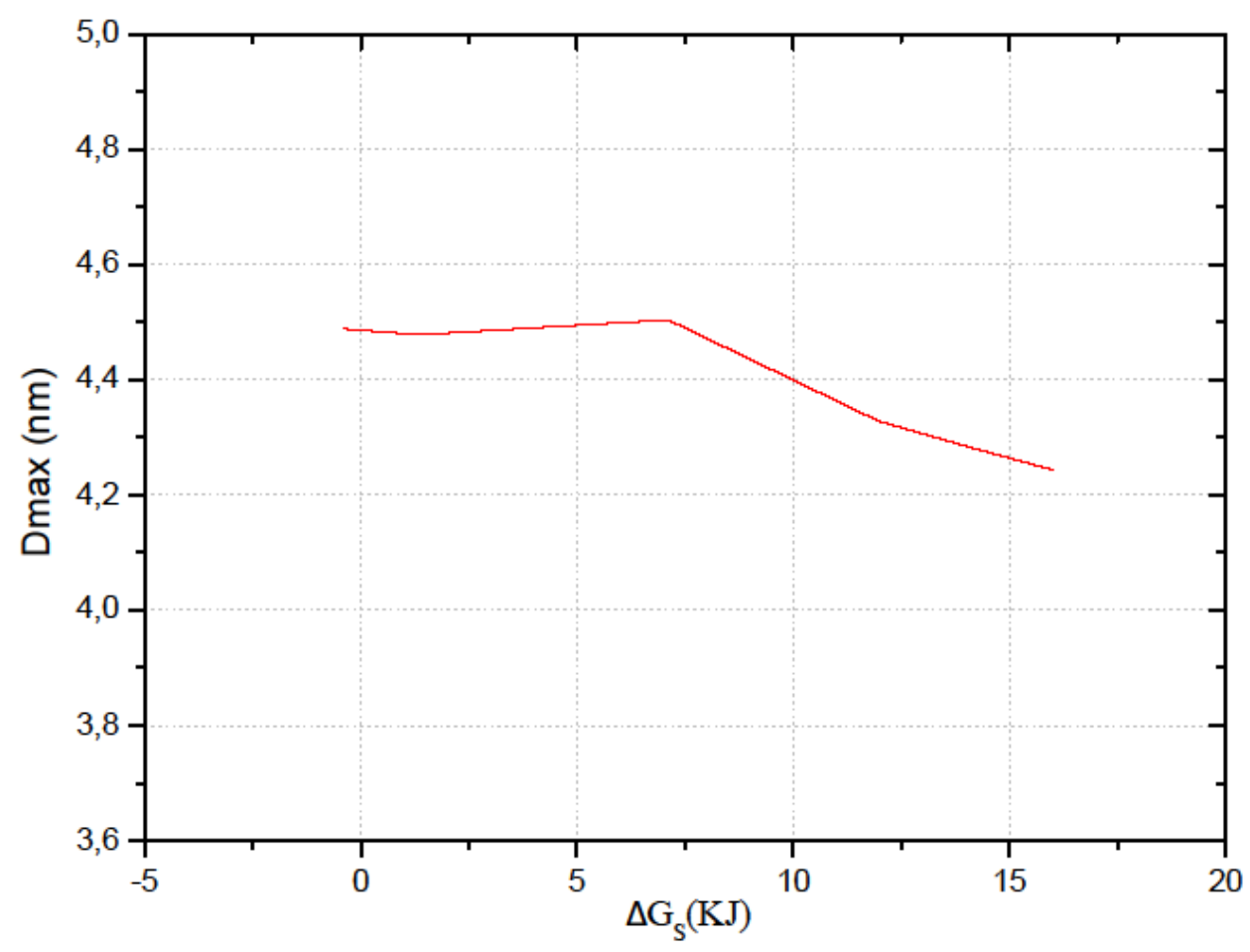

Figure 2: Calculated wood maximum swelling (i.e. swelling at 100\% $\mathrm{RH}$ ) in $\mathrm{nm}$, as a function of free energy of binding of the cation binding to the crystalline cellulose considering the hydrated ionic radius of the chaotropic ion considered. 


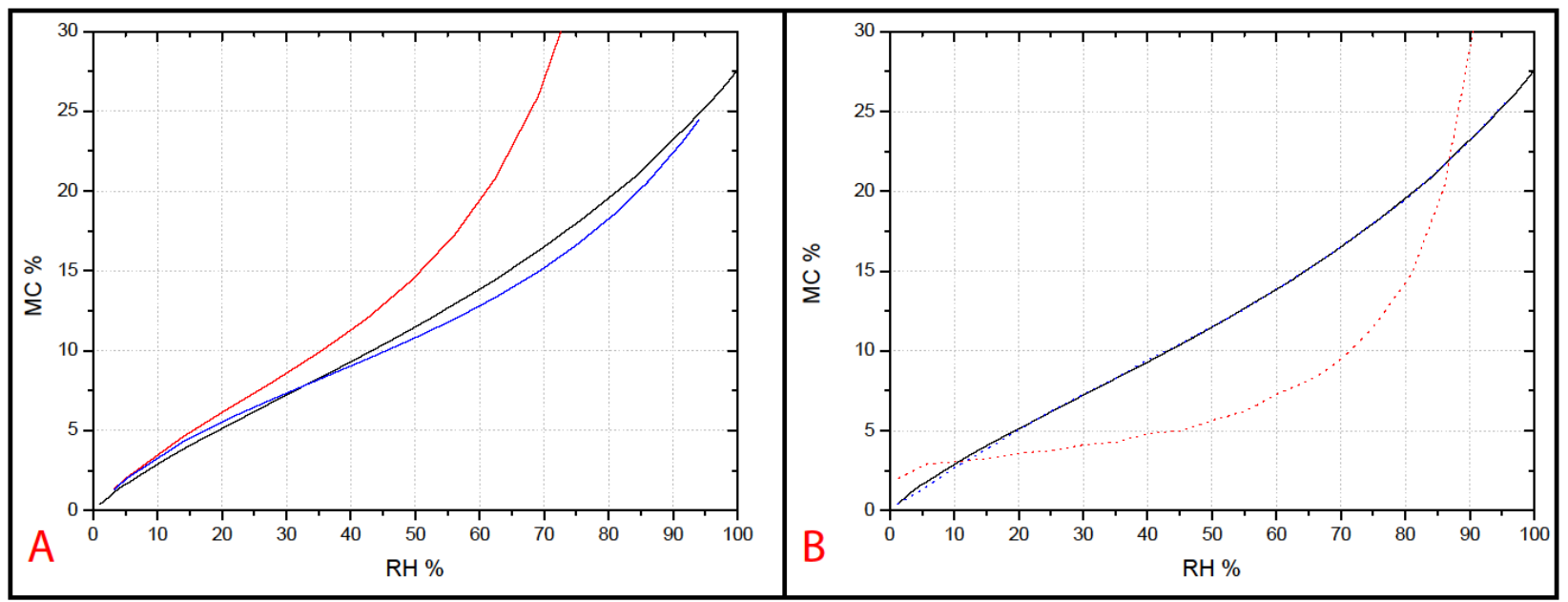

Figure 3: Wood Equation of State, in terms of moisture content MC as a function of relative humidity RH. a) comparison between modeling curve (black) and classical sorption models: BET (red) and Dent/HailwoodHorrobin (blue) b) results of fitting the classical curves (BET: dotted red, Dent/Hailwood-Horrobin: dotted blue) to the one calculated from the model (black).

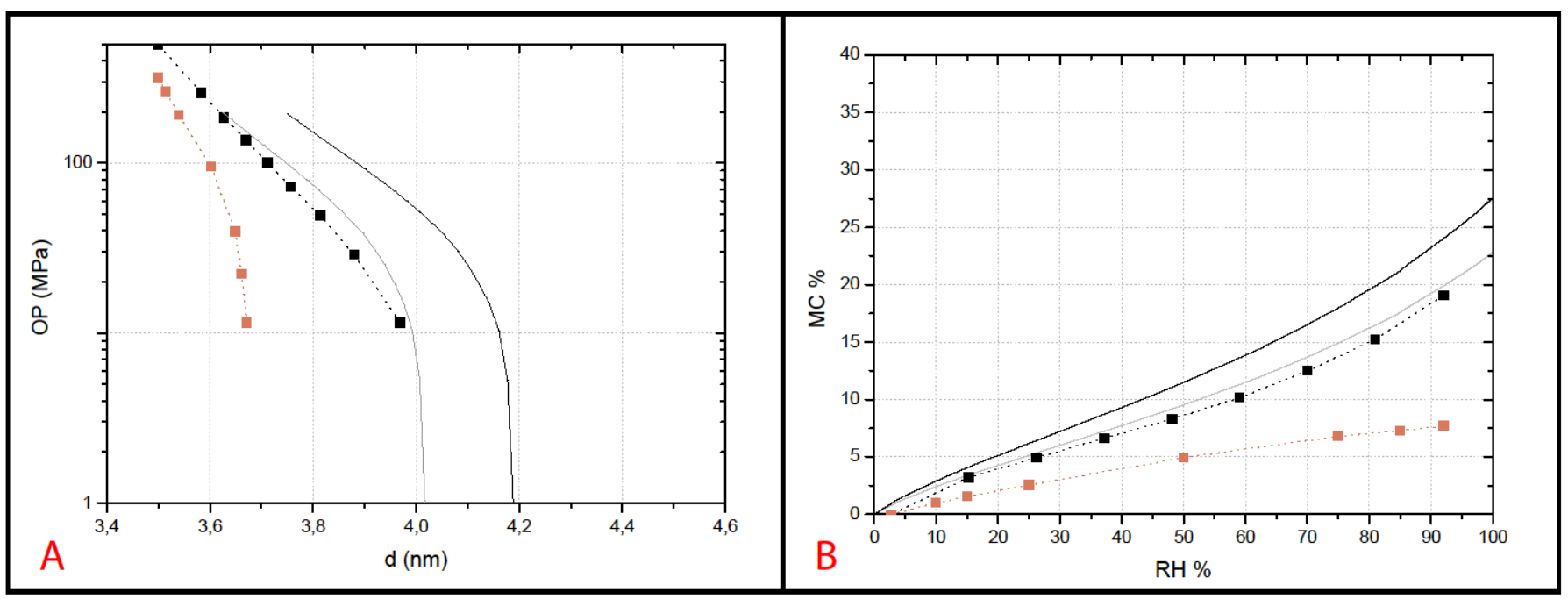

Figure 4: Equations of State, in terms of osmotic pressure as a function of intercrystalline distance (A) and in terms of moisture content MC as a function of relative humidity $R H(B)$ : wood EOS calculated from modeling (black line), wood EOS calculated from modeling at its lower limit (grey), wood sorption data from DSC dehydration experiment (black squares) and coir sorption data from DSC dehydration experiment (light brown). 


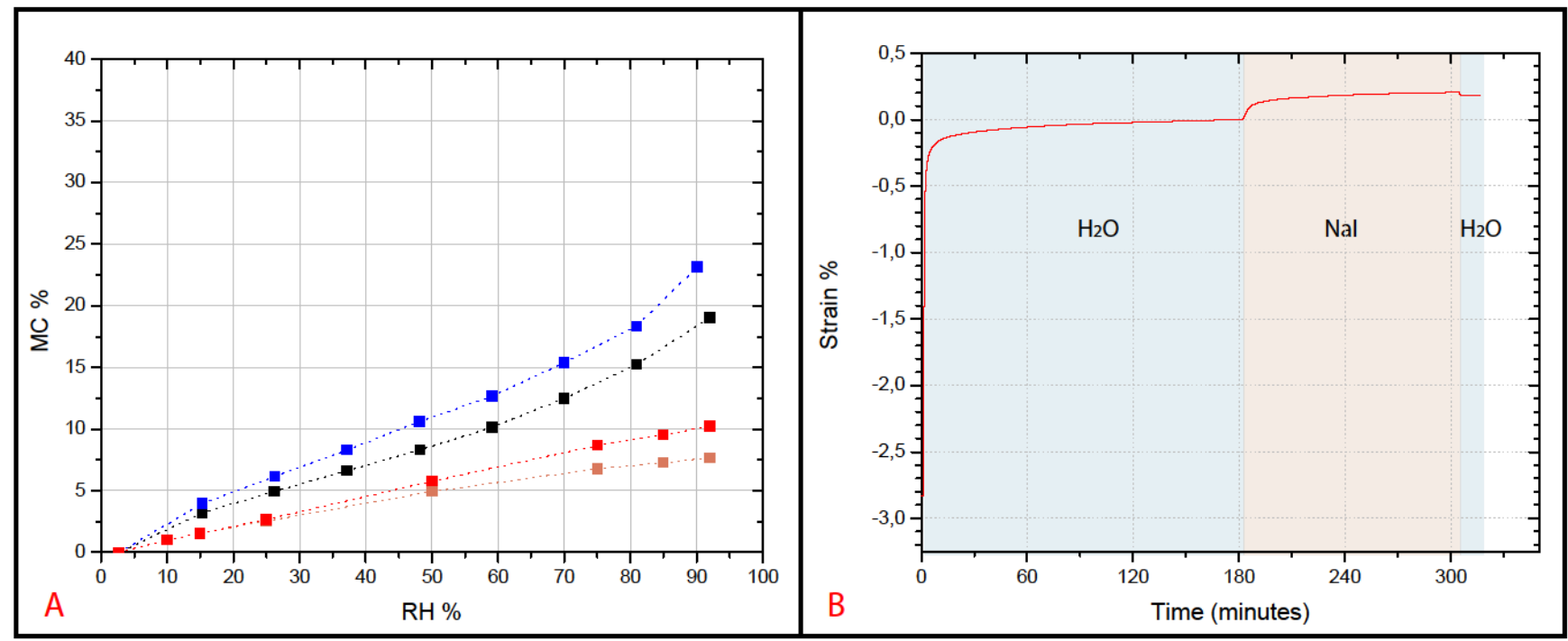

Figure 5: a) EOS expressed in terms of moisture content MC as a function of relative humidity $R H$, obtained from DSC dehydration experiments, for wood (black), wood pre-treated with a $4.70 \mathrm{M} \mathrm{NaI}$ solution (blue), coir (orange), coir pre-treated with a 4.70 M NaI solution (red). The same experimental EOS are reported in Appendix $A$ in the form OP vs d. b) Swelling profile of coir fibers sample, after wetting, impregnation with 4.0 NaI solution, and washing. 
Appendixes
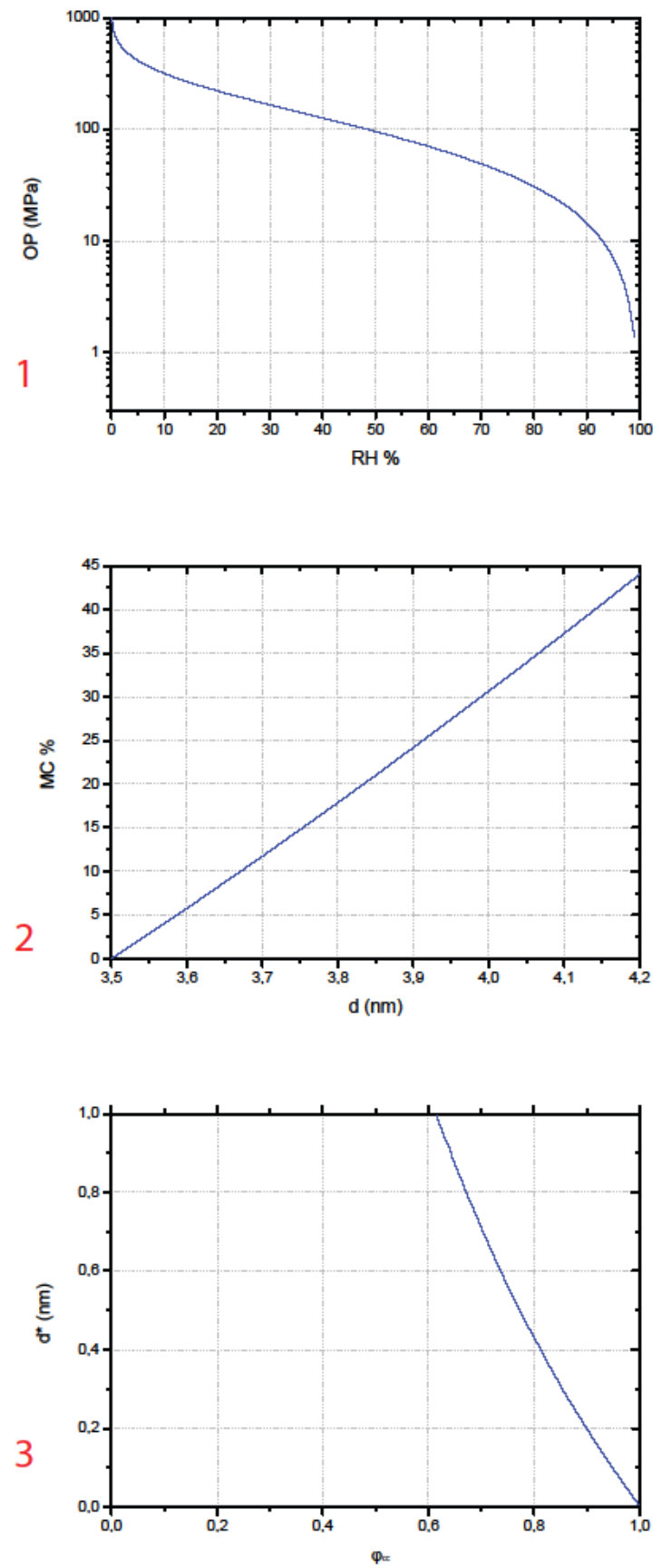


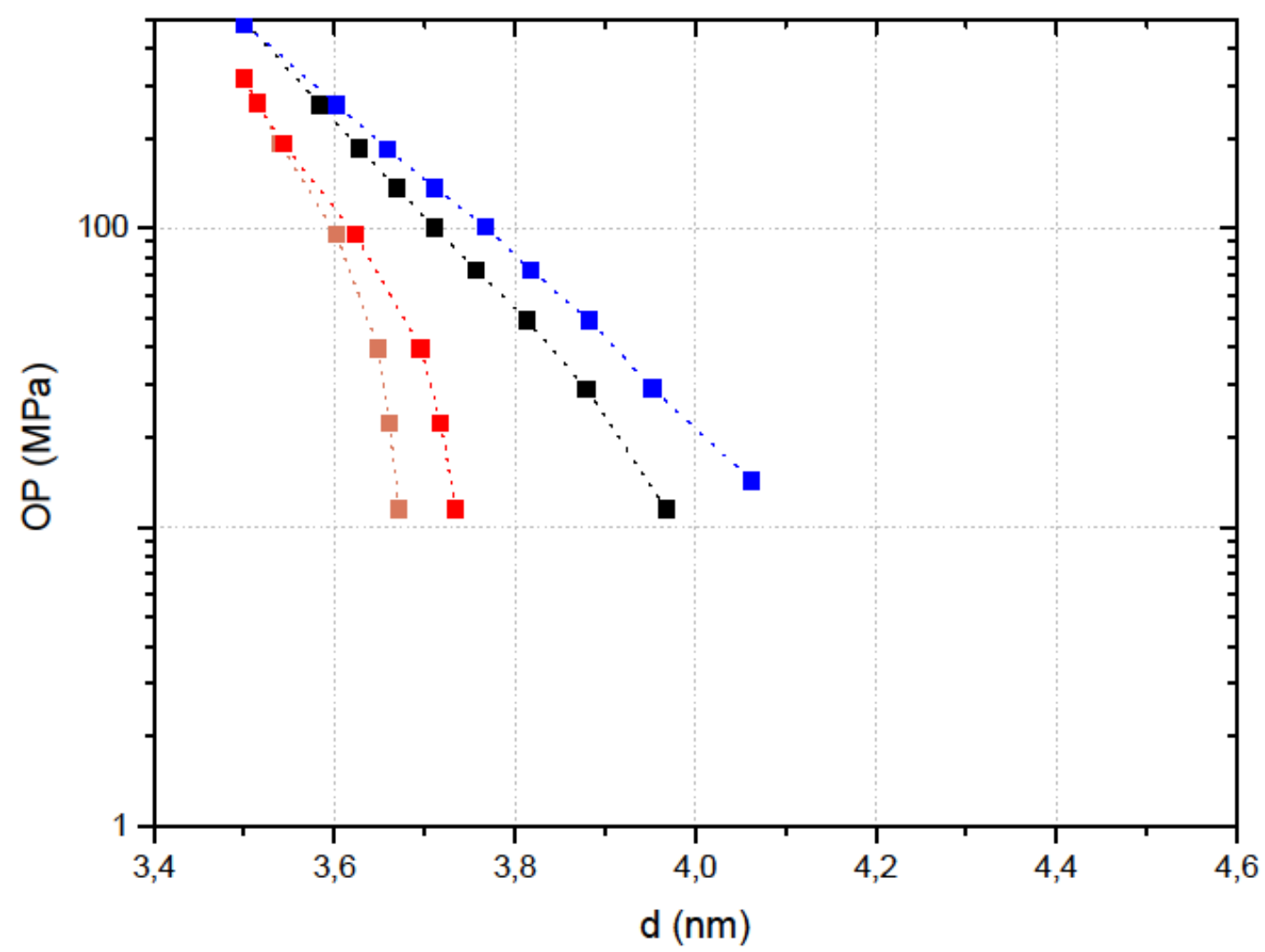

Table B.1: Calculated parameters for the sorption BET, Dent and H-H equations, for the case of spruce wood.

\begin{tabular}{|l|l|l|l|l|l|}
\hline \multicolumn{1}{|c|}{$M_{0}$} & \multicolumn{1}{|c|}{$M_{0}^{\prime}$} & \multicolumn{1}{c|}{$K_{1}$} & \multicolumn{1}{c|}{$K_{1}^{\prime}$} & \multicolumn{1}{c|}{$K_{2}$} & $K_{2}^{\prime}$ \\
\hline 8.70 & 8.70 & 6.23 & 5.15 & 0.712 & 0.712 \\
\hline
\end{tabular}

